\title{
Emotional modulation of the pupil in psychopathy: A test of the Response
}

\section{Modulation Hypothesis.}

Daniel T. Burley ${ }^{\mathrm{a}}$, Valerio Deriu ${ }^{\mathrm{a}}$, Rhys Masin ${ }^{\mathrm{a}}$, Nicola S. Gray ${ }^{\mathrm{b}, \mathrm{c}}$, Robert J. Snowden ${ }^{\mathrm{a}}$ aschool of Psychology, Cardiff University, 70 Park Place, Cardiff CF10 3AT, UK

${ }^{\mathrm{b}}$ Department of Psychology, Swansea University, Singleton Park, Swansea, SA2 8PP, UK ${ }^{\mathrm{c}}$ Abertawe Bro-Morgannwg University Health Board, Caswell Clinic, Glanrhyd Hospital, Tondu Road, Bridgend, CF31 4LN, UK.

Correspondence concerning this article should be addressed to:

Robert Snowden, School of Psychology, Cardiff University, Cardiff, CF10 3AT, UK. Contact: snowden@cardiff.ac.uk

Declarations of interest: none. 


\section{Abstract}

Some aspects of psychopathy may be underpinned by a deficit in processing emotional information, although there is evidence that this impairment only emerges when the affective cues are not central to ongoing goal-directed behaviour. However, this hypothesis has not been explored previously in relation to autonomic reactivity to emotional stimuli. The current study investigated this in a large $(N=174)$ community sample by examining changes in pupil diameter, a measure of autonomic nervous system activity, while participants viewed images that were either neutral in content or contained highly arousing/emotional content. Participants' attentional focus was manipulated across two tasks, such that participants either focused on whether the image contained emotional content (emotion-focus) or whether there were people present in the image (alternate-focus). Psychopathy was conceptualised via the Triarchic model of boldness, meanness and disinhibition. As expected, the arousing images caused greater pupil dilation compared to neutral images. However, the magnitude of this dilation was not moderated by any aspect of psychopathy regardless of the participant's attentional focus. It may be that reduced pupil reactivity to emotional stimuli is only expressed at high levels of psychopathy not normally found in community samples, or that participants with high traits of psychopathy in the community were still able to sufficiently attend to the emotional components of the images to overcome any autonomic deficits regardless of their attentional focus. Further research is needed to explore these possibilities.

Keywords: Pupillometry, psychopathy, Response modulation hypothesis, attention, emotion, attention, Triarchic model

\section{Abbreviations :}

RMH: Response Modulation Hypothesis; 
EMPR: Emotional modulation of the pupil response

TriPM : Triarchic Psychopathy Measure

\section{Introduction}

Psychopathy is understood as a multi-faceted personality disorder that is typically characterised by a combination of interpersonal-affective (e.g., a lack of empathy, callousness) and behavioural features (e.g., impulsivity, disinhibition), and is commonly associated with negative long-term outcomes (Hare and Neumann, 2008). Traditionally, the dominant theoretical perspectives conceptualised psychopathy as a unitary disorder with a single underlying impairment. Emotion-based theories of psychopathy have proposed that the behaviour exhibited by individuals with psychopathy is due to a fundamental emotional deficit, with the amygdala often implicated as central to this impairment (Blair et al., 2005, Kiehl, 2006). In support of this, behavioural, psychophysiological and neuroimaging research has demonstrated that individuals high in psychopathic traits show impaired emotional processing and responding (Brook et al., 2013). This includes deficient autonomic arousal in response to emotional stimuli (Burley et al., 2019, Benning et al., 2005a, Verona et al., 2004, Gillespie et al., 2019) which is an indicator of the sensitivity of underlying defensive and appetitive motivational systems (Lang and Bradley, 2010). However, the exact nature of this impairment has been unclear. A general emotional deficit perspective suggests that individuals high in psychopathy hold a blunted capacity for experiencing all emotions (Cleckley, 1976), while alternative accounts argue that psychopathy is associated with an impairment in processing only negative emotions. One of the most influential theories is the low-fear hypothesis (Lykken, 1957) that proposed that psychopathy is associated with a deficit in neurobiological 
systems responsive to fear - that is, individuals high in psychopathy show an impaired defensive motivational system leading to an insensitivity to fearful cues.

More recent theories have conceptualised psychopathy as a disorder of impaired general information processing. The Response Modulation Hypothesis (RMH; Newman and Lorenz, 2003) proposes that psychopathy is associated with a difficulty in the ability to shift attention to incorporate additional or later information peripheral to the individual's ongoing goaldirected behaviour. Importantly, as the hypothesised impairment is attentional in nature, the RMH model expects psychopathy to be associated with deficits in other domains (i.e., language/semantic processing, set-shifting) rather than emotional processing alone (BaskinSommers et al., 2011). Support for attentional accounts of psychopathy, such as the RMH, derives from studies that highlight that individuals high in psychopathic features show impairments outside of emotional processing alone (Hiatt and Newman, 2006) and evidence that psychopathic individuals show typical physiological responses to emotional stimuli when directed towards key threat-relevant emotional information (Baskin-Sommers et al., 2011, Larson et al., 2013, Newman et al., 2010). To illustrate, Newman et al. (2010) investigated fear-potentiated startle responses within adult offenders and manipulated their attention towards or away from threat-relevant cues. Participants were presented with coloured letters, where red letters were paired with electric shocks and green letters were not followed by a shock, and were asked to make a discrimination that focused them towards (threat-focused condition) or away from the colour of the letter (alternative-focus condition). Aversive white noise bursts were played to the participants during each condition and their startle responses were measured to these. Individuals high in psychopathy showed reduced potentiation of their startle response during the alternative-focus condition, but displayed typical responses under threat-focused conditions. This study suggests that abnormal attentional modulation may 
underlie emotional deficits observed for individuals high in psychopathy and that impairments can be alleviated if attention is explicitly directed towards the affective cues.

The RMH expects that individuals high in psychopathy would demonstrate universal processing deficits across all emotional valences providing that emotional information is presented peripherally to ongoing goal-directed attention. However, studies have repeatedly highlighted that individuals high in psychopathy demonstrate greater emotional processing impairments for negative rather than positive cues (Brook et al., 2013), which is particularly evident for psychophysiological responses (Burley et al., 2019, Esteller et al., 2016, Gillespie et al., 2019). This suggests the importance to integrate emotional and attentional processes when trying to understand the underlying impairments of psychopathy rather than exploring each process in isolation (Hamilton et al., 2015). Importantly, no study to our knowledge has explored predictions based on the RMH of psychopathy that focus on the role of attention to modulate autonomic reactivity to emotional cues.

Dual process models of psychopathy have challenged the unitary understanding of psychopathy and proposed separate genetic and neurobiological mechanisms underlying interpersonal-affective and impulsive-antisocial features of psychopathy (Patrick and Bernat, 2009, Fowles and Dindo, 2009, Yildirim and Derksen, 2015). It is suggested that the interpersonal-affective dimension of psychopathy reflects dispositional fearlessness caused by an insensitive defensive motivational system, while impulsive-antisocial dimensions relates to a vulnerability to externalising difficulties due to deficient functioning of fronto-cortical regions (Patrick and Bernat, 2009). Studies assessing autonomic reactivity have supported this distinction finding that interpersonal-affective psychopathy features, rather than impulsiveantisocial symptoms, were related to blunted responsivity to negative threat stimuli (Burley et al., 2019, Verona et al., 2004). Alternatively, the Triarchic model of psychopathy (Patrick et al., 2009) conceptualises interpersonal and affective features into two phenotypes, specifically 
boldness - this includes the nexus of social dominance, low stress reactivity and fearlessness and meanness - reflecting cruelty, lack of empathy, and excitement seeking. The third phenotype is termed disinhibition and relates to the externalising features of psychopathy including impulsiveness, irresponsibility, as well as dysregulated behaviour. The Triarchic model suggests that both boldness and meanness are disparate phenotypic manifestation of genetic disposition towards fearlessness (Patrick et al., 2009). Gillespie et al. (2019) examined the relationship between the Triarchic psychopathy scales and autonomic reactivity to static facial expressions as measured by pupillometry. It was found that only meanness was related to reduced pupil reactivity to affective expressions and this hypoactivity was in response to both negative and positive faces. Importantly, it is not clear what role attentional processes played within this emotional deficit as the participant's attention focus was not manipulated (i.e., towards/away from the salient emotional component). This is important to investigate further given previous evidence highlighting the situational specificity of emotional deficits in relation to psychopathy - that is, directing the individual towards salient affective cues reduced or alleviated the emotional deficit related to psychopathy.

The current study investigated whether manipulating participant's attention towards or away from important emotional cues affected the relationship between psychopathy symptoms and reactivity to emotional cues. Psychopathy was measured using the Triarchic Psychopathy Measure (TriPM, Patrick, 2010) along the dimensions of boldness, meanness and disinhibition. We measured changes in pupil diameter as an indicator of autonomic arousal in response to emotional images as pupillometry has previously been used to demonstrate that psychopathic features are linked to blunted autonomic reactivity (Burley et al., 2019, Gillespie et al., 2019). Pupil dilation in response to emotional stimuli reflects sympathetic activation (Bradley et al., 2017) mediated from the amygdala (Davis, 1992), suggesting that pupillary responses may provide a direct indicator of amygdala activation (Siegle et al., 2002, Koikegami and Yoshida, 
1953). There is also evidence that pupillometry is more reliable and time-sensitive measure of emotional arousal than alternative measures of sympathetic activity such as galvanic skin response (Bradley et al., 2017). Affective images were presented, instead of the facial expressions as used by Gillespie et al. (2019), as images lead to greater emotional modulation of the pupil compared to faces (Burley et al., 2017).

Consistent with Gillespie et al. (2019), we hypothesised that meanness specifically would be related to reduced pupil reactivity to negative stimuli. However, as explained by the $\mathrm{RMH}$, we hypothesised that this autonomic deficit to negative stimuli would only emerge when emotional cues were peripheral to the primary focus of goal-directed attention (i.e., participant's attentional focus was directed away from the emotional cues). In contrast, we hypothesised that no relationship between psychopathy symptoms and pupil reactivity to emotional stimuli would emerge when participants' attention was directed towards the salient emotional cues. A weaker or absent relationship was hypothesised between meanness and pupil reactivity to positive stimuli, given the stronger evidence for autonomic hypo-responsivity to negative stimuli (Brook et al., 2013), including pupil responsivity (Burley et al., 2019).

\section{Method}

\subsection{Participants}

Research using psychometric measures of psychopathy typically only produce relatively small effect sizes. Hence, we powered our study to be able to detect small to medium effect sizes $\left(f^{2}=.075\right)$ with a power of 0.8 at an alpha of .05 for a multiple regression analysis with three predictors, which results in a minimum sample of 150 people. However, we overrecruited given that data is often lost due to technical problems, etc. One-hundred and seventyfour participants were recruited from the School of Psychology participant panel at Cardiff University and advertisements on the official Cardiff University online forum for research 
participants and word of mouth from a student population in exchange for course credits or payment. Four participants were excluded for missing questionnaire or pupillometry data leaving a final sample of 170 participants (76 female, 94 male) with a mean age of 20.52 (S.D. $=2.75)$. The study was approved by the Research Ethics Committee at Cardiff University.

\subsection{Procedure}

The study procedures took place in a sound attenuated laboratory with dim lighting (approx. 5 lx). Participants gave written informed consent to all procedures. Participants took part in two pupillary tasks - the emotion-focus task and the alternative-focus task - and the order of these tasks was counter-balanced across participants. During the pupillometry tasks, each participant was seated approximately $60 \mathrm{~cm}$ away from the screen, underneath which a Tobii X2-60 eye tracking device was secured. A calibration procedure was conducted for each participant using a 5-point calibration screen. During calibration, the participant was instructed to view a moving target (red dot) as it moved sequentially between 5 points over the course of 10 seconds. The eye tracker then located the participant's pupils within an area of threedimensional space, allowing for small head movements to occur without interrupting measurement, and negating the need for a head rest. Following the tasks, the participant completed the TriPM in a quiet room by themselves.

\subsubsection{Pupillometry Task}

The pupillometry tasks were modelled on that of Burley et al. (2019). A schematic illustration of a trial is shown in Figure 1, which was the same across task conditions. Each trial began with a grey screen presented for 2000 ms. This blank screen included a fixation mark for the first 1000 ms. A test image was then presented for 2000 ms. Following target image presentation, participants were prompted to make a forced-choice decision by pressing an arrow key. This decision varied depending on the task. One task asked the participant whether each image was scary/happy or neutral (emotion-focus task), and the alternative task 
asked whether the image contained a person or not (alternate-focus task). The participant indicated their answer using the keyboard arrow keys with the left arrow reflecting an emotional image or an image with a person respectively, and the right arrow indicating the opposite. After the decision, a feedback screen (indicating a correct response or incorrect response) was shown for $500 \mathrm{~ms}$, followed by a blank recovery screen for $3700 \mathrm{~ms}$. Maximum decision time allowed was $2000 \mathrm{~ms}$, after which, if no response was detected, the feedback screen displayed "No response detected" and recovery occurred as in other trials. Each participant completed both the emotion-focus and the alternate-focus tasks with the order of these tasks counter-balanced across participants. Each task consisted of 30 trials and each took 5 minutes to complete. All screens preceding and following the images were luminancematched to the image set (approx. $150 \mathrm{~cd} / \mathrm{m}^{2}$ ).

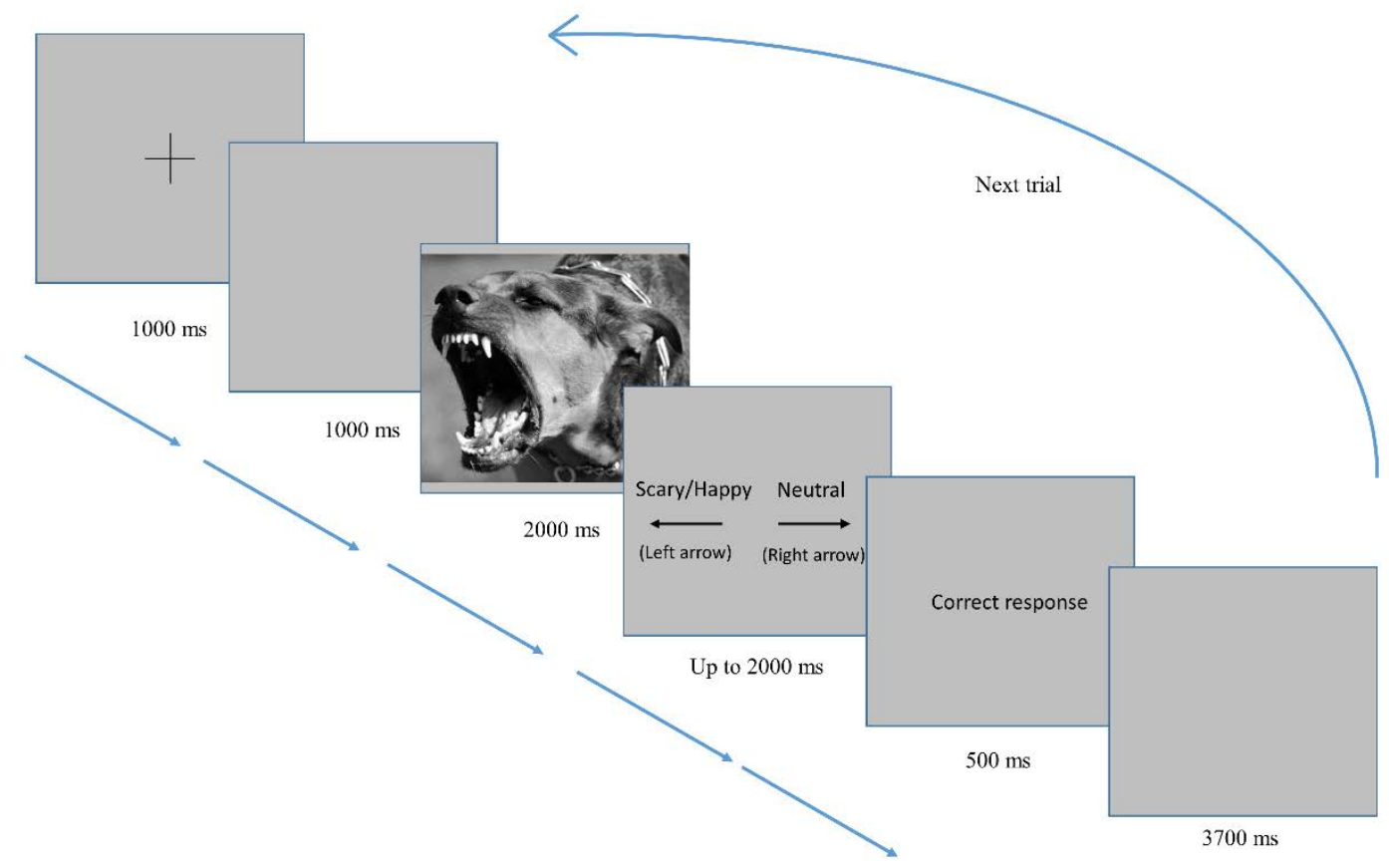

Figure 1. A schematic illustration of a trial from the pupillary tasks. 


\subsection{Materials and measures}

\subsubsection{Affective images}

Thirty images were presented from the International Affective Picture System (IAPS, Lang et al., 1997) based on the image set from Burley et al. (2019) ${ }^{1}$. These were presented in a random order. Ten were negative (mean valence/arousal based on IAPS ratings $=2.91,6.20$ ), 10 positive $(7.60,5.89)$, and 10 neutral images $(5.17,3.10)$. The images were a mix of social (e.g., assailant pointing a weapon, people laughing, a person on the phone) and non-social images (e.g. snarling dog, puppies, a garden door). Images were converted to greyscale and matched on dimensions of image contrast and luminance using Adobe Photoshop Elements 12.0.

\subsubsection{Psychopathy measure}

The Triarchic Psychopathy Measure (TriPM; Patrick, 2010) is a self-report measure consisting of 58 items (scored from 0 - 3, higher scores indicating higher psychopathic traits), which scores participants along the three dimensions of boldness, meanness and disinhibition. It has been shown to validly measure the construct of psychopathy when related to the PCL-R, the most commonly assessed measure of psychopathy (Venables et al., 2014). Good internal consistency and test-retest reliability have also been demonstrated for the TriPM (Blagov et al., 2016) and the current study reports good internal consistency ratings displayed in Table 1. Missing responses in the self-completed TriPM questionnaires were filled by pro-rating the average score for the relevant subscale (though these were $<1 \%$ of the scores). One participant's TriPM data was omitted for containing too many missing items ( $>10 \%)$.

${ }^{1}$ IAPS selected were: Negative 1301, 1304, 1525, 5973, 6231, 6242, 6250, 6263, 6370, 9901; Positive 1710, 2347, 4599, 4641, 7330, 8200, 8380, 8370, 8470, 8490; Neutral 2036, 2190, 2214, 2383, 2393, 2514, 2745.1, 2850, 2870, 5731. 


\subsection{Data Analysis}

The data cleaning methods are identical to that of Snowden et al. (2016). The pupil sample rate was $60 \mathrm{~Hz}$. An average of the left and right pupil diameter is reported in millimetres, and where data is missing for one eye the monocular estimate is taken. Any pupil diameter change of $\pm 0.38 \mathrm{~mm}$ to the previous data reading was attributed to random fluctuation and removed (Partala and Surakka, 2003). Data within $33.3 \mathrm{~ms}$ around these points were also removed to avoid anomalous readings. Pupillometry data was then smoothed using a SavitzkyGolay low-pass filter set to a span of 5 data readings. Missing data figures are reported after cleaning. A pre-stimulus onset 'baseline' period of 200 ms was calculated for each trial and subtracted from each subsequent data recording to establish a change score metric. The use of baseline-corrected data yields broadly consistent results to alternative methods including raw data and percentage change data (Attard-Johnson et al., 2019). The analysis was conducted using a purpose written script in Python using NumPy and Pandas extensions.

Data for each trial were omitted if less than $50 \%$ of data for the selected time window were available (19.7\% of all trials). Participant means for each emotional valence were only calculated if data were available for at least $50 \%$ of trials. Participants were also excluded if less than $50 \%$ of their data were available across all trials, and if their mean pupil response for a particular valence was identified as an outlier, i.e. outside the interval defined as three times the standard deviation for that valence. Three participants were excluded from the data analysis due to excessive missing data. Pupil size data were inspected for outliers and normality of distribution by visual inspection as recommended (Tabachnick and Fidell, 2007). No outliers were removed and the data approximated a normal distribution.

Mean baseline-corrected pupil size was determined in the "critical window" of 1000-2000 ms post-image presentation. It is typical to observe a pupillary constriction in response to the onset of visual naturalistic images despite overall luminance-matching to previous visual 
stimulus and is thought to reflect a parasympathetic response to an increase in visual contrast (Snowden et al., 2016; Bradley et al., 2017). Changes in pupil diameter in response to the affective content of stimuli are considered to reflect sympathetic activation, and this process begins around $500 \mathrm{~ms}$ following image-onset and is maximally visible once the parasympathetically-mediated pupil constriction is complete after approximately 800-1000 ms following image-onset (Bradley et al., 2017). An analysis window across 1000-2000 ms postimage onset therefore allows us to capture emotional modulation of the pupil in response to the images. Mean pupil diameter was calculated across all images of the same valence for both the emotion-focus task and the alternate-focus task for each participant. Split-half reliability estimates for mean pupil diameter using the Spearman-Brown correction revealed internal consistency coefficients of $r=.64-.72$ for the emotion-focus task and $r=.55-73$ for the alternative-focus task.

A 3x2 repeated measures ANOVA was performed to assess the emotion (negative, positive, neutral) by task-focus (emotion-focus, alternative-focus) effects on pupil diameter. We initially included participant gender and task-order (emotion-focus or alternate-focus task completed first) as between subjects factors, but there were no significant main effects or interactions involving these variables $(p s>.30)$ and so analyses were rerun with these variables omitted. Planned comparison t-tests were performed for pupil diameter between all valences for both tasks.

To test the effects of psychopathy on pupil responses specific to the emotional images across tasks for each individual, emotional modulation of the pupil response (EMPR) was calculated for each valence in each task condition (Burley et al., 2019). This was calculated by subtracting mean neutral pupil diameter (over 1000-2000 ms) from the mean fear and mean happy diameters respectively for each task. Larger EMPR value indicates greater emotional modulation of pupil diameter. To understand the effects of psychopathy on EMPR across tasks, 
we computed an ANCOVA including the factors of emotion (negative, positive) and task (emotion-focus, alternative-focus), with scores for Boldness, Meanness and Disinhibition entered as dimensional variables. We also ran follow-up partial correlations between each TriPM subscale and EMPR for each valence across each task to assess the specific association of each subscale. We applied a Bonferroni alpha correction for associations involving happy images given that the our a priori hypothesis was not specific regarding the exact effect of psychopathy across tasks on pupil responses to happy images (corrected $\alpha=.05 / 2$ task conditions $=.025)$.

\section{Results}

\subsection{Pupil response to images}

Figure 2 displays pupil diameter as a function of time since image-onset. The pupil constricted following presentation of all stimuli types (the pupillary light reflex) and then began to dilate at around $750 \mathrm{~ms}$. At this point the data from the different conditions begin to diverge showing the typical effects of emotion on pupil diameter (see Bradley et al., 2008, Snowden et al., 2016) to the end of the image presentation (2000 ms). To quantify the difference in pupil sizes due to image valence, we calculated the mean pupil diameter across 1000 - 2000 ms since image-onset (see Burley et al., 2019). Figure 2 shows that the pupil was more dilated in response to the emotional images than the neutral images (i.e., less negative). A repeated measures ANOVA with factors of emotion (fear, happy, neutral) and task-focus (emotionfocus, alternative-focus) was run to assess their effect on pupil diameter. There was a main effect of emotion, $F(2,338)=38.97, p<.001, \eta_{p}^{2}=.19 ; 95 \%$ CI $[.12, .26]$, a main effect of task-focus, $F(1,169)=12.43, p=.001, \eta_{\mathrm{p}}^{2}=.07 ; 95 \%$ CI $[.01, .15]$, but no significant interaction effect, $F(2,338)=0.26, p=.77, \eta_{\mathrm{p}}^{2}=.002 ; 95 \%$ CI $[.00, .02]$. 
To understand these main effects, we examined the difference between conditions using paired $t$-tests. Pupil diameter was larger overall in the Emotion-focus task $(-0.32 \mathrm{~mm}, \mathrm{SD}=0$ .25) compared to the Alternative-focus task $(-0.36 \mathrm{~mm}, \mathrm{SD}=0.25)$. Within each task, the fear and happy images produced greater pupil dilation than the neutral images ( $p s<.001$, $d s>.30$ ), and fear images led to larger pupil dilation than happy images in the alternative-focus condition $(p=.04, d=.13$ ). There was no difference between the fear and happy in the emotion-focus condition $(p=.29)$.
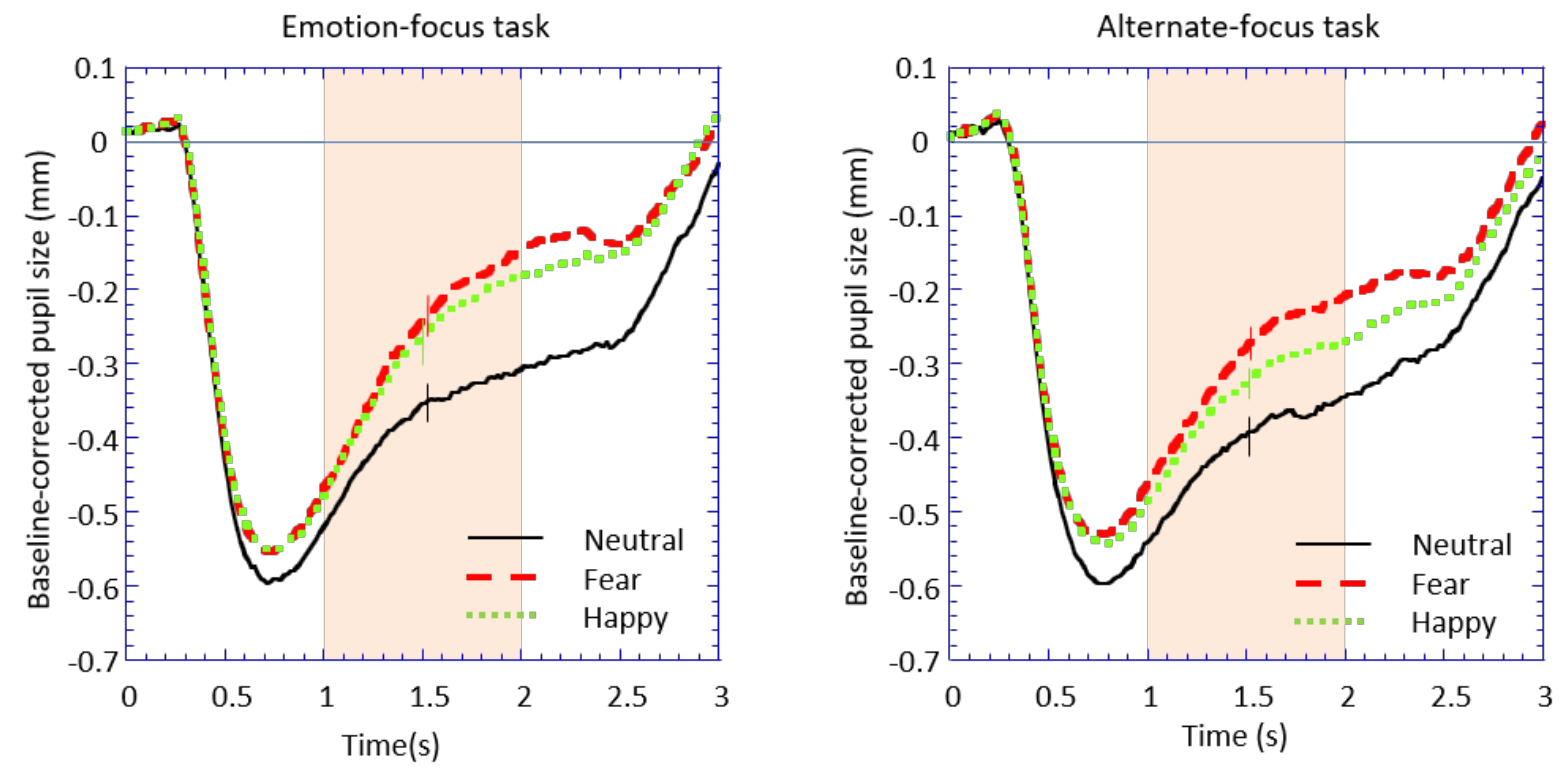

Figure 2. Pupil diameter in response to neutral (solid line) fear, (dashed line), and happy (dotted line) when participant attention is directed towards the emotional content of the image (emotion-focus) or alternatively towards whether there is a person in the image (alternatefocus). The image was presented from $0-2000 \mathrm{~ms}$ and the response window for statistical analysis was 1000 - 2000 ms (depicted by the shaded area). Error estimates are illustrated by an error bar ( \pm 1 SEM) at $1500 \mathrm{~ms}$ (centre of the response window). 


\subsection{Psychopathy}

Means, standard deviations, and correlations between the TriPM scales are displayed in Table 1. As can be seen in Figure 3, boldness displayed a normal distribution, but meanness and disinhibition were positively skewed, which was confirmed by a Shapiro-Wilk test of normality $(p s<.001)$. We therefore log transformed meanness and disinhibition for subsequent analyses.

Table 1. Scores and correlations for the Triarchic Psychopathy Measure. Figures in bold are the internal consistency of each scale.

\begin{tabular}{c|cccc}
\hline & Mean (SD) & 1 & 2 & 3 \\
\hline 1. Boldness & $30.9(8.1)$ & $.82^{* *}$ & $.36^{* *}$ & .06 \\
2. Meanness & $12.1(7.2)$ & & $.85^{* *}$ & $.48^{* *}$ \\
3. Disinhibition & $16.0(7.6)$ & & $.84^{* *}$ \\
\hline$* * p<.001$ & & &
\end{tabular}
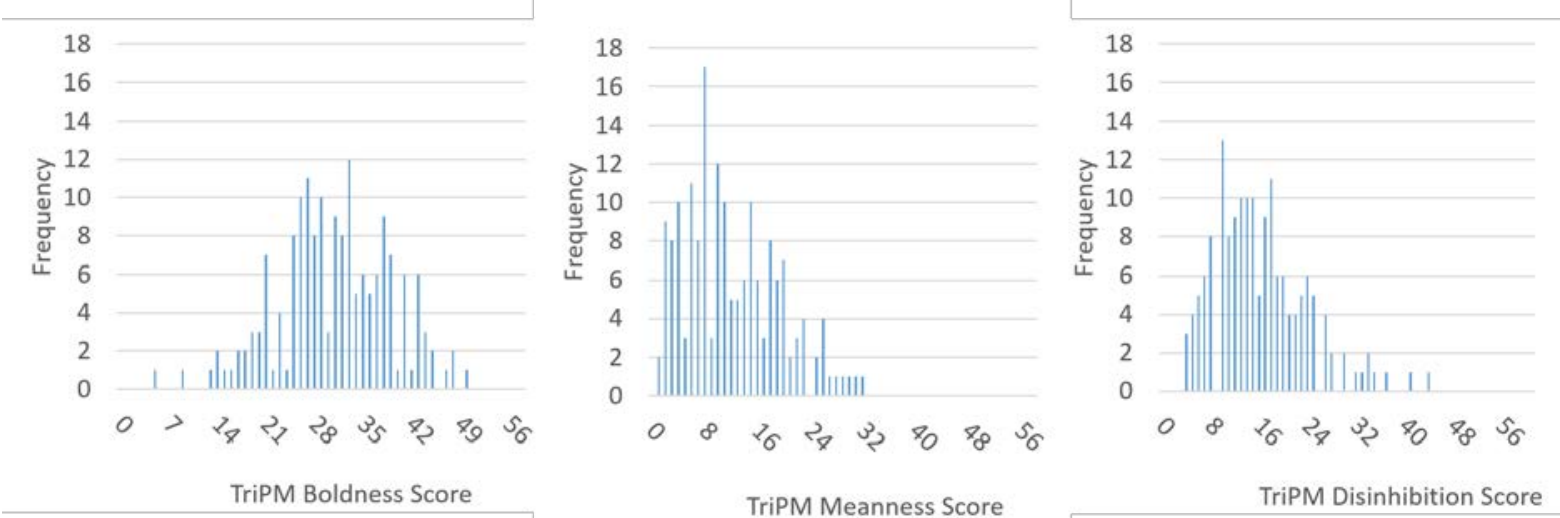
Figure 3. Frequency distribution for Triarchic Psychopathy Measure (TriPM) subscale scores across the sample.

An ANCOVA was computed to examine the effects of psychopathy, with factors of emotion (fear EMPR, happy EMPR) and task (emotion-focus, alternative-focus), and Boldness, Meanness and Disinhibition scores entered as dimensional variables. Table 2 indicates that Boldness, Meanness and Disinhibition showed no two-way interactions with either emotion or task and no 3-way interactions emerged for any of the subscales. This suggests that none of the Tri-PM subscales showed an effect on pupil response to either fearful or happy images.

As an additional check to explore the independent contribution of each Tri-PM subscale, we ran partial correlations between Boldness, Meanness and Disinhibition (while controlling for the remaining two subscales) and EMPR for each valence across tasks. As shown in Table 3, none of the Tri-PM subscales were uniquely related to EMPR for fearful or happy images across either task, apart from meanness being related to increased EMPR for the happy condition for the alternate-focus task $(p<.05)$, although this effect did not surpass significance when Bonferroni-corrections were applied for multiple comparisons.

Table 2. Analysis of covariance (ANCOVA) examining the effect of Tri-PM subscales on emotionally-modulated pupil response to fearful and happy images across emotion-focused and alternative-focused task conditions.

$$
F(1,166) \quad p \quad \eta_{\mathrm{p}}^{2} \quad(95 \% \mathrm{CI})
$$

Two-way interactions

Boldness $\mathrm{x}$ task 


\begin{tabular}{lcccc}
\hline Meanness x task & 0.86 & .35 & .01 & $(.00, .05)$ \\
Disinhibition x task & 0.02 & .89 & $<.001$ & $(.00, .01)$ \\
Boldness x emotion & 0.83 & .36 & .01 & $(.00, .05)$ \\
Meanness x emotion & 0.63 & .43 & $<.01$ & $(.00, .04)$ \\
Disinhibition x emotion & 0.91 & .34 & .01 & $(.00, .05)$ \\
Three-ways interactions & & & & \\
Boldness x task x emotion & 2.83 & .09 & .02 & $(.00, .07)$ \\
Meanness x task x emotion & 1.60 & .21 & .01 & $(.00, .06)$ \\
$\quad$ Disinhibition x task x emotion & 0.01 & .57 & $<.01$ & $(.00, .00)$ \\
\hline
\end{tabular}

Table 3. Partial correlations between Tri-PM subscale scores and emotion modulation of the pupil response (EMPR) in response to fearful and happy images for emotion-focus and alternative-focus task conditions.

\begin{tabular}{|c|c|c|c|c|c|c|c|c|}
\hline & \multicolumn{4}{|c|}{ Emotion-focus } & \multicolumn{4}{|c|}{ Alternative-focus } \\
\hline & \multicolumn{2}{|c|}{$\mathrm{EMPR}_{\mathrm{fear}}$} & \multicolumn{2}{|c|}{ EMPR $_{\text {happy }}$} & \multicolumn{2}{|c|}{$\mathrm{EMPR}_{\mathrm{fear}}$} & \multicolumn{2}{|c|}{ EMPR } \\
\hline & partial $r$ & $p$ & partial $r$ & $p$ & partial $r$ & $p$ & partial $r$ & $p$ \\
\hline Boldness & -.03 & .68 & .12 & .13 & -.05 & .54 & -.09 & .26 \\
\hline Meanness & .14 & .07 & .03 & .72 & .14 & .06 & $.17^{*}$ & $.03 *$ \\
\hline Disinhibition & -.07 & .34 & -.10 & .21 & -.05 & .52 & -.13 & .10 \\
\hline
\end{tabular}




\section{Discussion}

The study investigated whether manipulating participants' focus of attention - either towards or away from emotional content within images - affected the relationship between psychopathic traits and pupil reactivity to affective stimuli within a community population. It was expected that psychopathy, specifically meanness, would be related to blunted pupil responsivity to fear cues, but only when attention was directed away from the salient emotional information with no relationship emerging when attention was directed towards emotional information. That is, a deficit would only emerge when affective cues were peripheral to the primary focus of goal-directed attention, as proposed by the RMH. However, we found no evidence that psychopathic traits within a community sample as measured by the Triarchic Psychopathy model was related to reduced pupil responses regardless of the focus of attention.

\subsection{Attentional focus and pupil response to emotion}

The current study demonstrates that across both emotion-focus and alternate-focus tasks that pupil diameter is larger in response to emotional compared to neutral images, consistent with previous studies examining passive-viewing of emotional stimuli (Bradley et al., 2017, Snowden et al., 2016). Interestingly, we also observed that pupil diameter is larger during the emotion-focus than the alternate-focus task. Given that this effect is irrespective of image valence (including neutral images), it may be that larger pupil diameter is due to the increased complexity of making an emotional categorisation compared to a perceptual categorisation, given that the pupil increases with greater cognitive load (Beatty, 1982). Alternatively, the emotion-focus task may have led to increased emotional arousal that was reflected in larger pupil diameter throughout the task, whereas the alternative-focus task reflected a cognitive or perceptual appraisal task that led to lower emotional engagement. 


\subsection{Psychopathic traits and pupil response}

Our main aim of the study was to examine the $\mathrm{RMH}$, specifically that manipulating participant's attentional focus towards emotional features within stimuli would lead to a reduction or absence in the relationship between psychopathy and reduced autonomic responsivity. However, as no relationship between psychopathy and pupil responsivity to the emotional images was found, it is not possible to verify this hypothesis. Despite the current null findings, it is important that research continues to examine the RMH with psychopathy conceptualised as a dimensional construct given previous concerns that previous support for the $\mathrm{RMH}$ is based predominantly on extreme-groups design which may exaggerate group differences (Smith and Lilienfeld, 2015). Furthermore, our dimensional approach is broadly consistent with the Research Domain Criteria (RDoC) approach (Cuthbert, 2015), as we have attempted to examine threat-based and reward-based systems, alongside attentional processes, in relation to well-validated traits measures, which is important to try to understand the role of neurobiological processes in psychopathology (Patrick et al., 2013).

We found no evidence that psychopathic traits within a community population are associated with blunted autonomic reactivity to negative cues, regardless of attentional condition. The current results are in contrast to Burley et al. (2019) and Gillespie et al. (2019) who reported that interpersonal-affective features of psychopathy - specifically meanness in the latter study - were related to reduced pupil responses to a combination of negative visual stimuli (images, static facial expressions, dynamic facial expressions). It could be argued that this result may have occurred as participants were given a clear objective to either focus on the emotion within each image or to focus on whether there was a person present in the image, which led all participants to process (at least in part) the emotional features of the image overcoming any deficit associated with psychopathy. However, against this contention, Burley et al. (2017) similarly found no relationship between psychopathy traits - as measured by the 
Triarchic Psychopathy model - and pupil responses during passive-viewing of either images, or static and dynamic facial expressions, or listening to affective sound-clips where no clear objective was provided to participants. It may be that a stronger test of the RHM, and the possible revelation of psychopathy modulating the EMPR in community samples, will require attention to be diverted away from the image completely.

The present study and that of Burley et al. (2017) used a community-based population and failed to find an effect of psychopathy, whereas the studies of Burley et al. (2019) and Gillespie et al. (2019), which did identify an effect of psychopathy, recruited from an incarcerated population where more high extremes of psychopathy are likely to be found. We note that boldness and meanness scores in the current sample were similar with those reported by Gillespie et al. (2019) within a convicted sample. This links into a wider debate regarding whether findings in incarcerated populations in relation to psychopathy can be generalised to psychopathy as it is expressed in the community, whether in antisocial or non-antisocial form (Lilienfeld et al., 2015, Benning et al., 2018). It is not clear whether behavioural and physiological phenomena associated with psychopathy that has been identified within incarcerated populations, such as reduced pupil responses to affective stimuli, are only observed within criminal populations. This may occur due to higher autonomic responsivity to fear cues in relation to supposed 'successful' psychopathy perhaps indicative of increased ‘resilience’ (Ishikawa et al., 2001, Gao and Raine, 2010). Alternatively, the effects may be only expressed at higher levels of psychopathy regardless of setting (Benning et al., 2005b, Zimak et al., 2014). Further research is necessary to clarify this.

Interestingly, Esteller et al. (2016) reported that psychopathy traits, specifically boldness, within a community sample that showed similar levels of scores on the TriPM to the current sample was uniquely related to diminished fear-potentiated startle responses. This may indicate that measuring startle responses may be a more sensitive indicator of defensive 
motivational activity compared to measuring pupil responses to aversive stimuli, although pupillometry holds several advantages (no wires required, straight-forward, temporally sensitive indicator of ongoing autonomic activity). We note that our pupil diameter reliability estimates were relatively low compared to Burley et al. (2019), which limits the scope of our interpretation and may have contributed to the current null findings. Gillespie et al. (2019) have unfortunately not reported pupillometry reliability estimates for comparison. However, Burley et al. (2017) previously reported high reliability coefficients for pupillary data, yet likewise to the current study failed to find an effect of psychopathy on pupil response to emotional stimuli within a community sample.

\section{Conclusion}

We investigated possible emotion-attentional impairments in relation to psychopathy. It was found that psychopathic traits within a community population - as measured by the Triarchic Psychopathy model - were not related to reduced pupil reactivity to emotional images regardless of whether the participant's focus of attention was directed towards or away from the affective content. This suggests that reduced pupil reactivity to emotional stimuli does not emerge within community samples or only is expressed at high levels of psychopathy. In addition, the current finding fails to support or refute the $\mathrm{RMH}$, and further research is required to elucidate this finding.

\section{References:}

ATTARD-JOHNSON, J., CIARDHA, C. Ó. \& BINDEMANN, M. 2019. Comparing methods for the analysis of pupillary response. Behavior research methods, 51, 83-95.

BASKIN-SOMMERS, A. R., CURTIN, J. J. \& NEWMAN, J. P. 2011. Specifying the attentional selection that moderates the fearlessness of psychopathic offenders. Psychological Science, 22, 226 -234.

BEATTY, J. 1982. Task-evoked pupillary responses, processing load, and the structure of processing resources. Psychological bulletin, 91, 276.

BENNING, S. D., PATRICK, C. J. \& IACONO, W. G. 2005a. Psychopathy, startle blink modulation, and electrodermal reactivity in twin men. Psychophysiology, 42, 753-762. 
BENNING, S. D., PATRICK, C. J., SALEKIN, R. T. \& LEISTICO, A. R. 2005b. Convergent and discriminant validity of psychopathy factors assess via self-report. Assessment, 12, 270-289.

BENNING, S. D., VENABLES, N. C. \& HALL, J. R. 2018. Successful psychopathy. In C. J. Patrick (Ed.), Handbook of psychopathy (p. 585-608). The Guilford Press.

BLAGOV, P. S., PATRICK, C. J., OOST, K. M., GOODMAN, J. A. \& PUGH, A. T. 2016. Triarchic psychopathy measure: Validity in relation to normal-range traits, personality pathology, and psychological adjustment. Journal of Personality Disorders, 30, 71-81.

BLAIR, J., MITCHELL, D. \& BLAIR, K. 2005. The Psychopath: Emotion and the Brain, Blackwell Publishing.

BRADLEY, M. M., MICCOLI, L., ESCRIG, M. A. \& LANG, P. J. 2008. The pupil as a measure of emotional arousal and autonomic activation. Psychophysiology, 45, 602607.

BRADLEY, M. M., SAPIGAO, R. G. \& LANG, P. J. 2017. Sympathetic ANS modulation of pupil diameter in emotional scene perception: Effects of hedonic content, brightness, and contrast. Psychophysiology, 54, 1419-1435.

BROOK, M., BRIEMAN, C. L. \& KOSSON, D. S. 2013. Emotion processing in Psychopathy Checklist - assessed psychopathy: A review of the literature. Clinical Psychology Review, 33, 979-995.

BURLEY, D. T., GRAY, N. S. \& SNOWDEN, R. J. 2017. As far as the eye can see: relationship between psychopathic traits and pupil response to affective stimuli. PLoS One, 12.

BURLEY, D. T., GRAY, N. S. \& SNOWDEN, R. J. 2019. Emotional modulation of the pupil response in psychopathy. Personality Disorders: Theory, Research, and Treatment. 10, 365

CLECKLEY, H. 1976. The mask of sanity (5th Ed), St Louis, MO, Mosby.

CUTHBERT, B. N. 2015. Research Domain Criteria: toward future psychiatric nosologies. Dialogues in clinical neuroscience, 17, 89.

DAVIS, M. 1992. The role of the amygdala in fear-potentiated startle: Implications for animal models of anxiety. Trends in Pharmacological Sciences, 13.

ESTELLER, A., POY, R. \& MOLTO, J. 2016. Deficient aversive-potentiated startle and the triarchic model of psychopathy: The role of boldness. Biological Psychology, 117, 131140.

FOWLES, D. C. \& DINDO, L. 2009. Temperament and psychopathy: A dual-pathway model. Current Directions in Psychological Science, 18, 179-183.

GAO, Y. \& RAINE, A. 2010. Successful and unsuccessful psychopaths: A Neurobiological Model. Behavioral Sciences \& the Law, 28, 194-210.

GILlESPIE, S. M., ROTSHTEIN, P., CHAPMAN, H., BROWN, E., BEECH, A. R. \& MITCHELL, I. J. 2019. Pupil reactivity to emotional faces among convicted violent offenders: The role of psychopathic traits. Journal of abnormal psychology, 128, 622.

HAMILTON, R. K. B., RACER, K. H. \& NEWMAN, J. P. 2015. Impaired integration in psychopathy: A unified theory of psychopathic Dysfunction. Psychological Review, 122, 770-791.

HARE, R. D. \& NEUMANN, C. S. 2008. Psychopathy as a clinical and empirical construct. Annual Review of Clinical Psychology, 4, 217-246.

HIATT, K. D. \& NEWMAN, J. P. 2006. Understanding psychopathy: The cognitive side. In C. J. Patrick (Ed.), Handbook of psychopathy (p. 585-608). The Guilford Press.ISHIKAWA, S. S., RAINE, A., LENCZ, T., BIHRLE, S. \& LACASSE, L. 2001. Autonomic stress reactivity and executive functions in successful and unsuccessful 
criminal psychopaths from the community. Journal of Abnormal Psychology, 110, 423432.

KIEHL, K. A. 2006. A cognitive neuroscience perspective on psychopathy: Evidence for paralimbic system dysfunction. Psychiatry research, 142, 107-128.

KOIKEGAMI, H. \& YOSHIDA, K. 1953. Pupillary dilatation induced by stimulation of amygdaloid nuclei. Psychiatry and Clinical Neurosciences, 7, 109-126.

LANG, P. J. \& BRADLEY, M. M. 2010. Emotion and the motivational brain. Biological psychology, 84, 437-450.

LANG, P. J., BRADLEY, M. M. \& CUTHBERT, B. N. 1997. International Affective Picture System (IAPS): Technical manual and affective ratings. NIMH Center for the Study of Emotion and Attention.

LARSON, C. L., BASKIN-SOMMERS, A. R., STOUT, D. M., BALDERSTON, N. L., CURTIN, J. J., SCHULTZ, D. H., KIEHL, K. A. \& NEWMAN, J. P. 2013. The interplay of attention and emotion: top-down attention modulates amygdala activation in psychopathy. Cognitive Affective \& Behavioral Neuroscience, 13, 757-770.

LILIENFELD, S. O., WATTS, A. L. \& SMITH, S. F. 2015. Successful psychopathy: A scientific status report. Current Directions in Psychological Science, 24, 298-303.

LYKKEN, D. T. 1957. A study of anxiety in the sociopathic personality. Journal of Abnormal and Social Psychology, 55, 6-10.

NEWMAN, J. P., CURTIN, J. J., BERTSCH, J. D. \& BASKIN-SOMMERS, A. R. 2010. Attention moderates the fearlessness of psychopathic offenders. Biological Psychiatry, 67, 66-70.

NEWMAN, J. P. \& LORENZ, A. R. 2003. Response modulation and emotion processing: Implications for psychopathy and other dysregulatory psychopathology. In: DAVIDSON, R. J., SCHERER, K. \& GOLDSMITH, H. H. (eds.) Handbook of Affective Sciences. Oxford University Press.

PARTALA, T. \& SURAKKA, V. 2003. Pupil size variation as an indication of affective processing. International Journal of Human-Computer Studies, 59, 185-198.

PATRICK, C. J. 2010. Operationalizing the Triarchic Conceptualization of Psychopathy: Preliminary Description of Brief Scales for Assessment of Boldness, Meanness, and Disinhibition, Unpublished Manual

PATRICK, C. J. \& BERNAT, E. M. 2009. Neurobiology of psychopathy: A two process theory. In G. G. Berntson \& J. T. Cacioppo (Eds.), Handbook of neuroscience for the behavioral sciences, Vol. 2 (p. 1110-1131). John Wiley \& Sons Inc. https://doi.org/10.1002/9780470478509.neubb002057

PATRICK, C. J., FOWLES, D. C. \& KRUEGER, R. F. 2009. Triarchic conceptualization of psychopathy: Developmental origins of disinhibition, boldness, meanness. Development and Psychopathology, 21, 913-938.

PATRICK, C. J., VENABLES, N. C., YANCEY, J. R., HICKS, B. M., NELSON, L. D. \& KRAMER, M. D. 2013. A construct-network approach to bridging diagnostic and physiological domains: Application to assessment of externalizing psychopathology. Journal of Abnormal Psychology, 122, 902.

SIEGLE, G. J., STEINHAUER, S. R., THASE, M. E., STENGER, V. A. \& CARTER, C. S. 2002. Can’t shake that feeling: event-related fMRI assessment of sustained amygdala activity in response to emotional information in depressed individuals. Biological psychiatry, 51, 693-707.

SMITH, S. F. \& LILIENFELD, S. O. 2015. The response modulation hypothesis of psychopathy: A meta-analytic and narrative analysis. Psychological Bulletin, 141, 1145. 
SNOWDEN, R. J., O'FARRELL, K. R., BURLEY, D., ERICHSEN, J. T., NEWTON, N. V. \& GRAY, N. S. 2016. The pupil's response to affective pictures: Role of image duration, habituation, and viewing mode. Psychophysiology, 53, 1217-1223.

TABACHNICK, B. G. \& FIDELL, L. S. 2007. Using multivariate statistics (5th Ed), Boston, Pearson.

VENABLES, N. C., HALL, J. R. \& PATRICK, C. J. 2014. Differentiating psychopathy from antisocial personality disorder: A triarchic model perspective. Psychological Medicine, 44, 1005-1013.

VERONA, E., PATRICK, C. J., CURTIN, J. J., BRADLEY, M. M. \& LANG, P. J. 2004. Psychopathy and physiological response to emotionally evocative sounds. Journal of abnormal psychology, 113, 99.

YILDIRIM, B. O. \& DERKSEN, J. J. L. 2015. Clarifying the heterogeneity in psychopathic samples: Towards a new continuum of primary and secondary psychopathy. Aggression and Violent Behavior, 24, 9-41.

ZIMAK, E. H., SUHR, J. \& BOLINGER, E. M. 2014. Psychophysiological and neuropsychological characteristics of non-incarcerated adult males with higher levels of psychopathic personality traits. Journal of Psychopathology and Behavioral Assessment, 36, 542-554. 